\title{
Absence Makes the Mind Grow Fonder: Reconceptualizing Studies of Safety Learning in Translational Research on Anxiety
}

\author{
Hyein Cho ${ }^{1,2}$ • Ekaterina Likhtik ${ }^{3,4}$. Tracy A. Dennis-Tiwary ${ }^{2,1}$
}

Accepted: 19 November 2020 / Published online: 9 January 2021

(C) The Psychonomic Society, Inc. 2021

\begin{abstract}
Overgeneralized fear (OGF), or indiscriminate fear responses to signals of threat and nonthreat, is a well-studied cognitive mechanism in human anxiety. Anxiety-related OGF has been studied primarily through fear-learning paradigms and conceptualized as overly exaggerated learning of cues signaling imminent threat. However, the role of safety learning in OGF has not only received much less empirical attention but has been fundamentally conceptualized as learning about the absence of threat rather than the presence of safety. As a result, the relative contributions of exaggerated fear learning and weakened safety learning to anxiety-related OGF remain poorly understood, as do the potentially unique biological and behavioral underpinnings of safety learning. The present review outlines these gaps by, first, summarizing animal and human research on safety learning related to anxiety and OGF. Second, we outline innovations in methods to tease apart unique biological and behavioral contributions of safety learning to OGF. Lastly, we describe clinical and treatment implications of this framework for translational research relevant to human anxiety.
\end{abstract}

Keywords Safety learning and fear learning $\cdot$ Anxiety-related overgeneralized fear $\cdot$ Translational research $\cdot$ Clinical implications of safety learning

\section{Introduction}

Anxiety disorders are among the most common and costly mental illnesses, affecting more than $30 \%$ of individuals during their lifetime (Eisenberg \& Lipson, 2017; Kessler et al., 2012; Kroenke et al., 2007). Moreover, approximately $30 \%$ of the patients diagnosed with an anxiety disorder do not respond to current "gold-standard" treatments, such as cognitive behavioral therapy (CBT; Beck, 1964) or selective serotonin reuptake inhibitors (Bystritsky, 2006), demonstrating significant gaps in our understanding of the mechanisms that underlie the etiology and remediation of anxiety.

Tracy A. Dennis-Tiwary

tracy.dennis@hunter.cuny.edu

1 Department of Psychology, The Graduate Center, The City University of New York, New York, NY, USA

2 Department of Psychology, Hunter College, The City University of New York, 695 Park Avenue, HN636, New York, NY 10065, USA

3 Department of Biology, Hunter College, The City University of New York, New York, NY, USA

4 Department of Biology, The Graduate Center, The City University of New York, New York, NY, USA
Overgeneralized fear (OGF), or indiscriminate fear responses to threat and nonthreat alike, particularly when they are perceptually similar (Duits et al., 2015; Lissek \& Grillon, 2015; Lissek et al., 2008, Lissek et al., 2014b; Sep et al., 2019), is one purported symptom of anxiety that has been a target of both clinical and neuroscience research. OGF is hypothesized to result from the diminished ability to perceptually discriminate signals of an imminent threat from signals that indicate safety or the absence of such impending threat, which leads to stimulus generalization (i.e., cue-driven response) or context generalization (i.e., context-driven response; Dunsmoor \& Paz, 2015; Dymond et al., 2015; El-Bar et al., 2017; Haaker et al., 2015; Lissek et al., 2005; Lissek et al., 2014b).

Indeed, studies with humans and animals showed that perceptual thresholds widen in instances of OGF (i.e., the sensitivity to detect and discriminate among different sensory stimuli is altered, impairing perceptual thresholds, thus inducing OGF) and that high-anxiety individuals demonstrate shallower sensory discrimination curves (i.e., increased generalization of fear-related stimuli; Aizenberg \& Geffen, 2013; Dunsmoor \& Paz, 2015; Schechtman et al., 2010; Wood et al., 2017). In line with these findings, a meta-analysis revealed that individuals with high-trait anxiety show higher OGF 
across stimulus type (i.e., unlike in healthy controls, OGF toward cue vs. context or novel cue vs. safety cue were all positively correlated with anxious personality traits), further suggesting that OGF is a potential mechanistic pathway that may increase vulnerability of the high-trait anxious group for anxiety disorders (Sep et al., 2019).

The high relevance of OGF to clinical anxiety has propelled the field to examine closely the properties and mechanisms of anxiety-related OGF, primarily from the perspective of fear learning in studies of animals (Aizenberg \& Geffen, 2013; Han et al., 2008; Likhtik et al., 2014) and humans (Dunsmoor \& Paz, 2015; Lissek et al., 2008; Sep et al., 2019). Research on associative learning about threat through fear learning paradigms documents anxiety-related impairments across species, such as exaggerated fear acquisition, failure to inhibit the fear response, and delayed extinction learning (Davis et al., 2000; Eysenck, 1979; Raber et al., 2019; for a review, see Lissek et al., 2005).

However, a growing body of research suggests that disruptions in safety learning may be a distinct and complementary mechanism underlying anxiety-related OGF. Learned safety has been considered a parallel of "conditioned inhibition" of fear (Pavlov, 1927; Pollak, Monje, \& Lubec, 2010; Rescorla, 1967), in which the fear response to a conditioned stimulus is blocked as a result of learning safety cues. Despite evidence for unique neural circuitry and behavioral effects of safety learning shown largely via animal research (Kong et al., 2014; Ostroff et al., 2010; Rogan, Leon, Perez, \& Kandel, 2005), in humans it has been studied predominantly as a secondary component of fear learning (e.g., fear discrimination learning; Christianson et al., 2012; Dymond et al., 2015; Lissek et al., 2005; Lonsdorf et al., 2017). In other words, in human literature safety learning often is characterized as a response to cues that signal relative safety (i.e., the absence of a threat stimulus) compared with cues that overtly signal threat (Lissek et al., 2010). While important, this approach does not directly measure learning about cues that signal the explicit presence of safety.

To illustrate the distinction between safety cues that indicate the absence of threat versus those that indicate the explicit presence of safety, consider the real-world example of walking through a dark park on a cold night to reach a friend's house. In the park, there are cues suggesting threat (e.g., a loud rustling noise behind the trees) and cues suggesting safety in the form of neutralized or absent threat (e.g., the rustling is accompanied by chirping sounds or the sight of wings, indicating that a "presumably" nonthreatening bird is causing the rustling). In contrast, cues of explicit safety include signs that you are approaching the friend's house (e.g., seeing the porch light outside your friend's house or hearing the sounds of friendly voices), which provides warmth, shelter, and friendship - that is, the presence of safety. Despite their conceptual and operational independence, these two types of cues have been largely conflated in the human and animal literature as a safety cue. As a result, the relative contributions of exaggerated fear learning (e.g., rustling is always perceived as threatening, regardless of its origin) and weakened safety learning (e.g., seeing the light on the porch does not ease anxiety) to anxiety-related OGF remain poorly understood, as do the unique biological and behavioral underpinnings of safety learning.

In this review, we first summarize research on anxietyrelated OGF in both animal and human models, focusing on underlying neural mechanisms. Next, we review evidence that safety learning is disrupted in anxiety and outline methodological challenges in isolating the mechanisms of safety learning that are distinct from those of fear learning. We then conceptualize safety learning as an active form of learning with direct clinical applications relevant to anxiety-related OGF and argue that we need methodological innovations to understand the role of safety learning in OGF to advance our understanding of mechanisms and clinical implications of OGF in anxiety. Throughout the review, we prioritize translational research that compares animal and human models of safety learning to identify future research avenues on cross-species mechanisms underlying anxiety-related OGF.

\section{Anxiety-related OGF}

OGF is characterized by indiscriminate and excessive fear responses that extend to items, contexts, and events that are not necessarily associated with threat (e.g., people, situations; Craske et al., 2009; Dymond et al., 2015; Lissek, 2012; Pittig et al., 2018). For example, an anxious individual may experience an aversive event (e.g., being criticized harshly for a verbal presentation), and this experience may lead the individual to infer that any cues related to or resembling this event (e.g., all forms of public speaking) are threatening and dangerous (Craske et al., 2009; Dymond et al., 2015). Furthermore, one may generalize the fear response not only to an entire category (e.g., public speaking) but to the items and places associated with the aversive event or object (e.g., a podium, auditorium; Dymond et al., 2015). When OGF is rigid and broad based on perceptual, semantic, and associative similarities, it will typically fuel both heightened behavioral avoidance and cognitive inflexibilities, such as threat hypervigilance, which in turn exacerbate anxiety (Mathews \& MacLeod, 2005; Mogg et al., 2004).

While fear in the face of potential danger is an evolutionarily adaptive response, sustained and indiscriminate reactivity to nonthreatening stimuli is inappropriate and can interfere with the ability to perform daily activities (Asok et al., 2018; Indovina et al., 2011; Likhtik \& Paz, 2015). Furthermore, heightened sensitivity toward threat and safety for an extended 
period of time may maintain or exacerbate a persistent anxiety phenotype (Bar-Haim et al., 2007; Dunsmoor \& Paz, 2015; Lissek et al., 2014b).

Fear conditioning paradigms have been predominantly used in research in animals and humans to assess anxiety-related OGF (Lissek et al., 2014b; Lonsdorf et al., 2017). These paradigms allow for the examination of acquisition, expression, extinction of emotional learning (e.g., threat-related memory), discrimination learning (discussed below), and the underlying mechanisms associated with these processes (Duits et al., 2015; Dunsmoor \& Paz, 2015; Hajcak, Castille, et al., 2009; Hajcak, Dunning, \& Foti, 2009; Lissek \& Grillon, 2015; Phelps \& LeDoux, 2005; Weinberger, 2007). Fear conditioning is a type of associative learning that links an aversive, negatively valenced "unconditioned" stimulus (US) with a neutral stimulus that initially elicits no emotions but which, through pairing with the US, is converted to a "conditioned" stimulus (CS) that elicits an aversive reaction even when presented alone upon completion of learning (i.e., conditioned response; Pavlov, 1927). These paradigms have been widely employed to identify basic neurobiological underpinnings of conditioned emotional learning and how these processes are aberrant in human anxiety. For example, anxiety is robustly associated with increased amygdala activity during acquisition and expression of learned fear and diminished modulation of the fear response via the ventromedial prefrontal cortex (vmPFC), which also is involved in extinction (e.g., inhibition of fear response; Indovina et al., 2011; Lissek et al., 2014a; Milad et al., 2005; Meyer \& Bucci, 2014; Meyer et al., 2019; Phelps et al., 2004; Quirk et al., 2006; Iordanova et al., 2007).

Fear discrimination paradigms have been of particular importance in the study of OGF in humans and animals, because they provide metrics of how fear learning generalizes across cues and contexts based on characteristics, such as perceptual, semantic, and associative similarities (Asok et al., 2018; Lissek et al., 2008; Sep et al., 2019; Struyf et al., 2015, 2017; see Dymond et al., 2015 for a review on anxietyrelated OGF). Fear discrimination paradigms include two CS types: the CS+ and $\mathrm{CS}^{-}$. The $\mathrm{CS}+$ is always presented before an aversive US (e.g., a shock), whereas the CS- is always presented without a subsequent US (see Lonsdorf et al., 2017 for a review of current methods in fear learning and the use of fear discrimination in human research). Thus, only the CS+ is predictive of a threat and the CS- signals relative nonthreat. Anxiety has been associated with the reduced ability to discriminate these two cues, which has become a "gold standard" measure of OGF (Lissek et al., 2014b).

\section{Neurobiological Basis of OGF}

Cross-species research using fear discrimination paradigms has linked OGF to disruptions in medial prefrontal cortex (mPFC)- amygdala circuitry (Asok et al., 2018; Cha et al., 2014; Grunfeld \& Likhtik, 2018; Likhtik et al., 2014; Likhtik \& Paz, 2015; Lissek et al., 2014a), including interactions with the hippocampus, auditory, and entorhinal cortices (Besnard, \& Sahay, 2016; Fani et al., 2012; Lissek et al., 2014a; Liu et al., 2020; O'Reilly \& Rudy, 2001; Padilla-Coreano et al., 2016; Rozeske et al., 2018; Wigestrand et al., 2017; Zelikowsky et al., 2014). These neural structures are considered to underlie fear discrimination, because they are implicated in key related processes, including encoding of information about CS-US relationships, perceptual discrimination of threat versus nonthreat stimuli, production, and inhibition of the fear response (see Figure 1 for predicted outcome for OGF associated with structures in the fear circuit). The interactions among these regions play an imperative role in fear discrimination as the basolateral complex of the amygdala (BLA) responds to emotionally salient information (Grosso et al., 2018; Letzkus et al., 2011; Phelps \& LeDoux, 2005), the hippocampus and entorhinal cortex encode and provide contextual information (Jin \& Maren, 2015; Maren et al., 1997; Maren \& Fanselow, 1996; Maren, Phan, \& Liberzon, 2013; O'Reilly \& Rudy, 2001), the sensory cortices encode the incoming cues (Aizenberg \& Geffen, 2013; Concina et al., 2018), and the mPFC communicates with a plethora of regions, regulating downstream responses (Cha et al., 2014; Do-Monte et al., 2015; Fani et al., 2012; Grunfeld \& Likhtik, 2018; Rozeske et al., 2018) via top-down selective encoding of threat-related information and subsequent action selection determined by input from the amygdala (i.e., BLA), hippocampus, and sensory cortices among others (Grunfeld \& Likhtik, 2018; Rozeske et al., 2018; Weinberger, 2007).

Pharmacology, in vivo physiology, and circuit manipulation during behavior all point to the involvement of this circuitry in OGF (Burgos-Robles et al., 2017; Giustino \& Maren, 2015; Karalis et al., 2016; Klavir et al., 2013, 2017; Likhtik et al., 2014; Moscarello \& Maren, 2018; Seidenbecher et al., 2003; Stujenske, Likhtik, Topiwala, \& Gordon, 2014). In rodents, increased mPFC-BLA communication, measured as increased synchrony in mPFC-BLA theta-range oscillations (4$12 \mathrm{~Hz}$, peaking at 4-6 Hz), and increased inhibition of amygdala output are associated with effective discrimination of threat from nonthreat and underlies appropriate response selection (i.e., expression or suppression of fear response) to a given stimulus (Izadmehr et al., 2017; Klavir et al., 2013; Likhtik et al., 2014). Specifically, during fear discrimination recall, mPFC-BLA synchrony is associated with better discrimination between cues signaling threat and nonthreat, whereas less synchrony is associated with greater OGF (Likhtik et al., 2014). Likewise, within the BLA, better discrimination is associated with increases in BLA fast gamma oscillations $(70-120 \mathrm{~Hz})$, which are considered to reflect activity of fast-spiking inhibitory interneurons (Buzsáki \& Wang, 2012; Likhtik et al., 2014; Stujenske et al., 2014). 
In this target circuit associated with OGF, the hippocampus has been studied in terms of its role in OGF-related contextual learning (Besnard, \& Sahay, 2016; Lissek et al., 2014a; McHugh et al., 2007; O'Reilly \& Rudy, 2001; Redondo et al., 2014), and its role in fear generalization has been shown via brain damage or lesion studies (Bucci et al., 2002; Wild \& Blampied, 1972). Specifically, the dentate gyrus of the hippocampus encodes the context where threat occurred, transferring this information to the hippocampal $\mathrm{CA} 3$ and $\mathrm{CA} 1$ regions. In this view, contexts encoded via different hippocampal engrams distinguish similar contextual representation [e.g., episodic contents of aversive (CS+) and nonaversive (CS -) memories during fear discrimination] to regulate and constrain OGF (Besnard, \& Sahay, 2016; Wild \& Blampied, 1972). The hippocampus encodes all cues as part of the context. During fear discrimination conditioning, the $\mathrm{CS}+$ is the most salient aspect of the entire context (e.g., as opposed to CS- or the absence of any cue), which yields contextual association with the CS+ dominating the context engram (Guo et al., 2018; Wang et al., 2012).

Hippocampal communication with the $\mathrm{mPFC}$ and with the BLA aids in transferring contextual information within this network (Girardeau et al., 2017, Kim \& Cho, 2017; Moita et al., 2004; Seidenbecher et al., 2003), whereas communication between the MPFC and BLA is more related to transferring information about the cue (Ito \& Morozov, 2019; Karalis et al., 2016; Klavir et al., 2013; Likhtik et al., 2014; Taub et al., 2018). Interactions within this network then leads to inhibition of inappropriate fear response to nonthreat (Lissek et al., 2014).
Research with humans has identified neural mechanisms underlying OGF similar to those documented in research with animals, such as the role of the $\mathrm{mPFC}$ in regulating and inhibiting fear responses and the functional and structural connectivity in amygdala-mPFC circuitry. Likewise, initial evaluation of and failure to distinguish threat from nonthreat is associated with deficient recruitment of the ventromedial PFC (vmPFC; Cha et al., 2014; Greenberg et al., 2013; Lissek et al., 2014a). In a study using a generalization task combined with functional magnetic resonance imaging (fMRI), patients diagnosed with generalized anxiety disorder (GAD) were asked to discriminate a threat (CS+ paired with shock) from nonthreat (unpaired $\mathrm{CS}^{-}$) and other items [i.e., Generalization stimuli (GS)] that perceptually resembled CS+ and CS- across a spectrum (e.g., shapes gradually changing in size towards or away from the target stimulus; Greenberg et al., 2013). Compared with healthy controls, patients displayed reduced activity of the vmPFC (e.g., "flatter" neural gradients; Cha et al., 2014). Behaviorally, ratings that were administered to assess subjective ratings on the likelihood of receiving the shocks reflected that the ratings for nonthreat stimuli were generalized (i.e., a less steep response gradient for GS from the CS+; Cha et al., 2014). This suggests diminished fear discrimination across stimuli given that fear inhibition is expected to occur to explicitly nonthreatening cues. Concordantly, MRI measures of vmPFC, including vmPFC thickness, functional and structural connectivity/integrity within the cortico-limbic system (i.e., the fear circuit) independently predicted individual variability in vmPFC activation (i.e., responsiveness) during a generalization task, further emphasizing the role of this

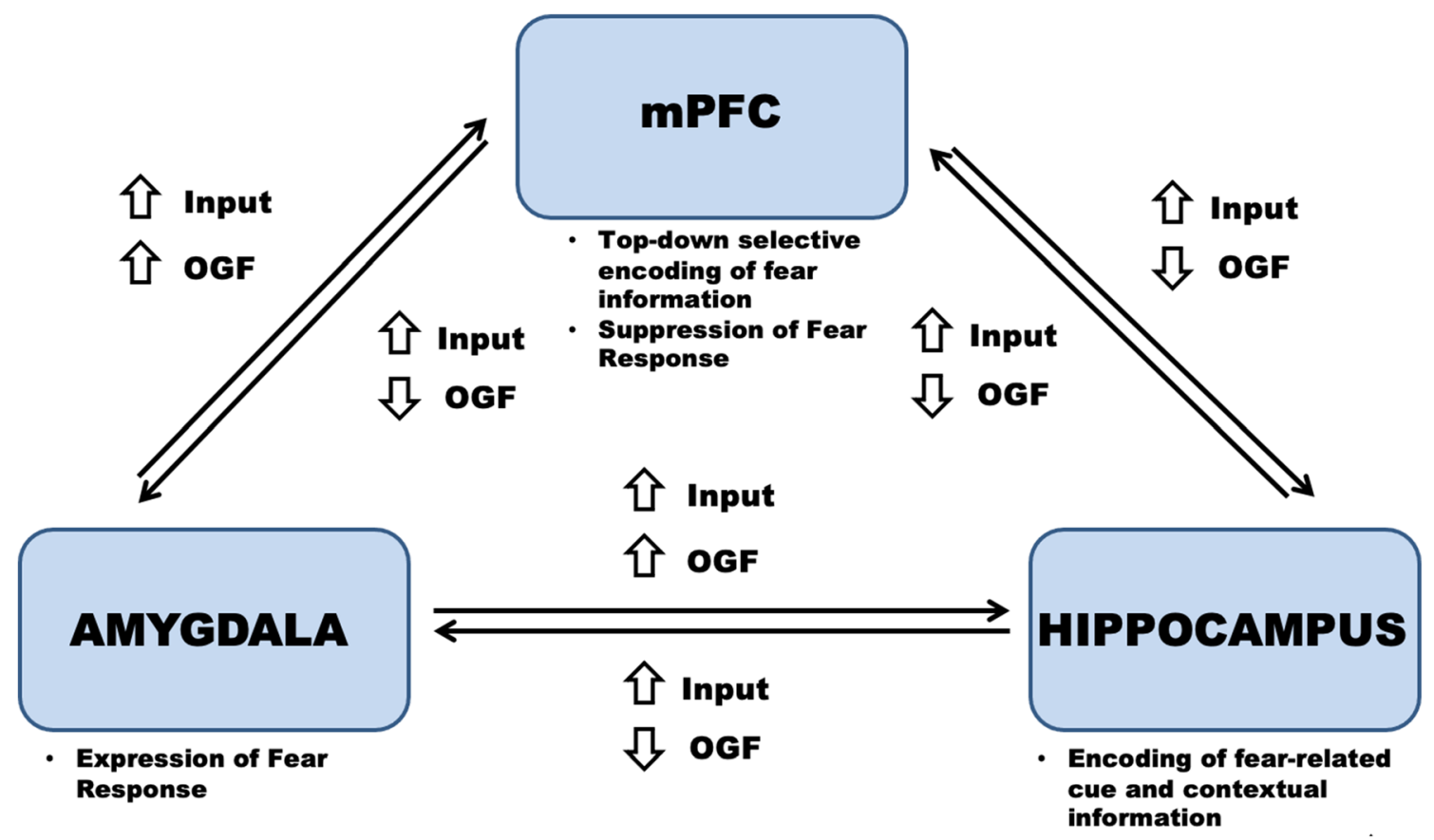

Fig. 1 Neural circuitry associated with predicted outcomes of increased input from a particular structure on OGF. 
region in regulating OGF (Cha et al., 2014). In addition to the neural substrates outlined above, other regions also play an important role in threat-safety discrimination. For example, recordings show that the ventrolateral periaqueductal grey (vlPAG) processes positive aversive prediction errors (unexpected delivery of shock) during fear conditioning and discriminative fear conditioning (Johansen et al., 2010; Ozawa et al., 2017; McNally \& Cole, 2006; Walker, Wright, Jhou, \& McDannald, 2020). Neural firing in the vlPAG tracks expectations of CS-US co-occurrence and its inhibition results in generalization, which is likely communicated to the amygdala via feedback loops (Walker et al., 2020; Ozawa et al., 2017; Johansen et al., 2010). Likewise, the orbitofrontal cortex (e.g., ventrolateral $\mathrm{OFC}$ ) has been associated with threat-safety discrimination learning, such that OFC inhibition before retrieval results in fear generalization (Sarlitto, Foilb, \& Christianson, 2018; Ray et al., 2018). Interestingly, dopaminergic cells in the ventral tegmental area (VTA) were shown to signal negative aversive prediction error (no delivery of expected shock), as is the case when a threat-associated cue undergoes extinction, with the VTA projection to nucleus accumbens (NAc) playing a role in this type of learning (Luo et al., 2018). Furthermore, the NAc was shown to process discrimination of an explicit safety cue from a cue that has a more probabilistic outcome (Ray et al., 2020), suggesting that the NAc plays a role in determining the comparative value of stimuli. In support of this idea, the NAc was shown to partake in processing relief cues that signal the end of an aversive event rather explicit safety cue itself (Mohammadi, Bergado-Acosta, \& Fendt, 2014). Zona incerta is another region that mediates fear discrimination, and its inhibition resulted in increased fear generalization (Venkataraman et al., 2019).

Neurophysiological studies provide further, noninvasive assessments of anxiety-related OGF. For example, adults diagnosed with GAD compared with healthy controls showed reduced visual discrimination and responsiveness to aversive compared with nonaversive stimuli measured via EEGderived event-related potentials (ERPs; the N170 and errorrelated positivity), both linked to generators in medial prefrontal cortical structures (Buzzell et al., 2017; Denefrio, Myruski, Mennin, \& Dennis-Tiwary, 2019). Other studies utilized the late positive potential (LPP), which is an ERP component that is enhanced during elaborative processing of emotionally salient or motivationally significant visual stimuli (Cuthbert et al., 2000; Hajcak, Castille, et al., 2009; Hajcak, Dunning, et al., 2009). In the case of fear learning paradigms, the CS+, if learning is successful, is more salient and motivationally significant relative to $\mathrm{CS}-$ and thus should be associated with LPPs of greater magnitude, which several studies have documented (Böcker et al., 2004; Bublatzky \& Schupp, 2012, but see Nelson et al., 2015). The LPP, thus, provides a sensitive index of disruptions in discriminative (threat-safety) learning related to anxiety.

\section{Safety learning and OGF}

Growing evidence suggests that in addition to fear learning, safety learning is disrupted in anxiety in ways that could contribute to OGF (Jovanovic et al., 2012; Lis et al., 2020). In a recent meta-analysis of 44 studies examining fear learning in anxiety, people diagnosed with anxiety disorders, compared with healthy controls, indicated both elevated responses to nonthreat cues during the acquisition phase of a learning task (measured as higher skin conductance responses to the CS-, which signals nonthreat) and elevated responses to the CS+ during extinction (Duits et al., 2015). This pattern also has been documented in individuals with high-trait anxiety compared with healthy controls (Gazendam et al., 2013; Haddad et al., 2012; Lis et al., 2020). Yet, the relative contributions of disruptions in fear versus safety learning to OGF remains poorly understood in part because the study of neural underpinnings of safety learning is in its infancy, particularly in humans (Meyer et al., 2019; Pollak, Rogan, et al., 2010; for a review, see Kong et al., 2014). Neurobiological evidence from rodents suggests that safety learning may differ from fear learning at the levels of the synapse (e.g., differential modification of spine structure in the lateral amygdala; Ostroff et al., 2010), genetic modifications, including unique expression of mircoRNA in the amygdala (Ronovsky et al., 2019), neural circuitry (e.g., posterior insular cortex, ventral hippocampal neurons; Christianson et al., 2008, 2011; Meyer et al., 2019, respectively), molecular expression (e.g., BDNF expression; Pollak et al., 2008), and individual-cell physiology, where a safety cue activates a different subset of cells from a fear cue in the amygdala (Sangha et al., 2013) and in the prefrontal cortical regions (Sangha et al., 2014).

One important step in clarifying these mechanisms is to examine the methods used to assess safety learning. For example, in fear discrimination learning paradigms, the nonthreat cue (CS-) is conceptualized as a cue predicting the absence of the US or aversive stimulus and is contrasted with the threat cue (CS+). Therefore, using this method, the CS- signals the absence of threat rather than the explicit presence of safety. One paradigm that tests explicit, active forms of safety learning must be a single-cued paradigm - that is, it must include a single CS- cue that is explicitly unpaired with the US and indicates explicit safety without the coexisting CS+ and CS- cues (Christianson et al., 2012; Lovibond et al., 2013; Pavlov, 1927; Pollak, Monje, et al., 2010; Rescorla, 1967; Rogan et al., 2005). Another way of studying safety learning is to present a CS+, which when presented alone predicts an aversive outcome, but if this CS+ is accompanied by a safety cue, it predicts the lack of an aversive outcome, indicating that when $\mathrm{CS}+$ is combined with a $\mathrm{CS}-$, it operates as a safety cue (Christianson et al., 2012; Meyer et al., 2019; Pollak et al., 2008; Sangha et al., 2013). 
While both single-cued and discrimination methods are widely used in fear learning research, a recent review of cross-species fear learning methodologies reveal that distinct methods are used to study fear acquisition in animals versus human research. The majority of human studies utilize fear discrimination to assess fear learning because it provides higher statistical power via the within-subject design (i.e., comparing CS+ vs. CS- response within the same subject), allowing researchers to avoid obtaining a separate control group for comparisons (Duits et al., 2015; Lissek et al., 2005; Lonsdorf et al., 2017). However, there are inherent limitations in the design in terms of the role of the CS- (e.g., interpretation and validity for the inhibitory conditioning to the $\mathrm{CS}^{-}$) using fear discrimination, whereby it may introduce confounds such as carryover effect from the previously presented $\mathrm{CS}+$, or perceptual ambiguity in the cues, indicating threat compared to the relative safety cues (Lonsdorf et al., 2017).

Unlike human studies, the majority of animal studies investigating fear acquisition mechanisms employ a betweensubjects approach, single-cued fear learning paradigms, which involves only one stimulus per study session, establishing a clear, explicit association between CS and US (Lonsdorf et al., 2017). Given this design, larger sample sizes are required, presenting practical barriers. However, the benefit of this approach is that it allows for a somewhat clearer differentiation between safety and fear-learning mechanisms. Indeed, a metaanalysis revealed that differences between patients and healthy controls in fear learning were not apparent when a discriminative fear-learning protocol was used but instead emerged only when single-cue paradigms were employed (Lissek et al., 2005).

Notably, the role that contextual features play in fear and safety cue processing is an important aspect that distinguishes these two approaches. Background conditioning of the context occurs when an explicit cue is paired with an outcome, such as a shock, whereas foreground conditioning occurs when the context itself serves as the explicit CS (Gould \& Bevins, 2012; Huckleberry et al., 2016). In the case of discrimination learning, background conditioning of the context occurs during both cues without any foreground conditioning, because no cue is paired with the context itself. However, during single-cue paradigms, background conditioning occurs during single-cue fear conditioning, whereas foreground and background conditioning occurs during the safety conditioning given that the aversive cue is presented in the context and the cue signals its absence. Given that different neural processing underlies these two types of learning, using singlecued paradigms allows for a distinct window into the effects of context on fear and safety learning.

Using single-cued fear and safety learning paradigms, research with rodents shows that explicit safety learning may induce more than just simple inhibition of the fear response and actually may promote positive affect (Kong et al., 2014; Ostroff et al., 2010; Pollak et al., 2008; Pollak, Monje, et al., 2010; Rogan et al., 2005; Sangha et al., 2013). Furthermore, given the importance of the safety cue as an active silencer of context-evoked (foreground conditioned) fear, it has the potential to be an effective conditioned inhibitor across contexts (Huckleberry et al., 2016). That is, safety learning induces unique learning outcomes that can be behaviorally observed (e.g., an increase in exploration behavior in a novel environment that is naturally aversive to the animal or decrease in the defensive freezing response to threat) and that are distinct from those of fear learning (Lovibond et al., 2013; Pollak et al., 2008; Pollak, Monje, et al., 2010; Rogan et al., 2005). This highlights the potentially unique clinical relevance of cued safety learning, such as producing anxiolytic effects and bolstering behavioral flexibility (Christianson et al., 2012; Kong et al., 2014; Meyer et al., 2019; Pollak et al., 2008; Rogan et al., 2005).

Rodent research with single-cued paradigms further suggests that the presence of safety signals may have motivational and rewarding properties. Early work in rats showed that when a tone that was previously paired with a shock was presented with a safety cue that signaled the absence of shock, animals were motivated to bar press for food, whereas the bar pressing response was inhibited when the $\mathrm{CS}+$ was presented alone (Hendry, 1967). In another study, safety-conditioned mice were compared with control mice that were simply exposed to the CS (e.g., tone; Rogan et al., 2005). After conditioning, the two groups of mice were given a "conditioned place preference test." The test revealed that the safetyconditioned mice preferred a compartment with the safety CS (i.e., while the tone was playing), whereas the controls had no preference, indicating that the safety CS had a positive valence (i.e., "CS preference"; Rogan et al., 2005). In support of this interpretation, recordings in the BLA show that a group of "safety-cue responsive" cells becomes active when a conditioned safety tone is played together with a fear tone to indicate trials without an aversive outcome. A subset of these "safety-responsive" cells also has been shown to be active during a cue that signals reward (Sangha et al., 2013).

Safety learning research in humans is in its nascent stage. To our knowledge, only one study to date used independent active single-cue fear and safety learning paradigms between subjects (i.e., instead of fear discrimination with the relative CS+ and $\mathrm{CS}^{-}$) to examine their unique, direct effects on functioning of amygdala-cortical circuitry and autonomic reactivity to fear cues (Pollak, Rogan, et al., 2010). Here, the authors found that fear-conditioned (paired CS) participants showed pupillary dilation (i.e., indicating emotional arousal and autonomic activation) in response to the threatening CS, whereas the safety-conditioned (unpaired CS) participants showed pupillary constriction to the safety CS. Using fMRI and diffusion tensor imaging during a single training session, they further 
documented that safety learning is associated with dampened activity in the amygdala and increased activity in the dorsolateral PFC, indicating increased top-down regulation of emotion. Using a within-subject comparison, one study showed that a unique neural circuitry was associated with safety learning compared with fear learning demonstrated via functional MRI (Meyer et al., 2019). Subjects were trained and tested on all conditions with different cues (i.e., threat, safety, and compound). The results showed that hippocampal and dorsal anterior cingulate cortex functional connectivity, but not hippocampal and anterior ventromedial prefrontal cortex or hippocampal and basolateral amygdala connectivity, was associated with safety (i.e., fear inhibition), marking importance of unique neural networks in safety learning compared with fear learning (Meyer et al., 2019).

There remain many gaps in our understanding of the neural mechanisms underlying the link between the presence of safety signals and OGF. However, one imaging study showed that the presence of safety signals was associated with reduced amygdala and enhanced dorsolateral PFC activity (Pollak, Rogan, et al., 2010), suggesting that safety signals may ameliorate disruptions in threat detection and inhibition related to this circuit and that characterize OGF (Christianson et al., 2012; Lissek et al., 2014b).

Taken together, prior research suggests that safety learning is disrupted in anxiety and OGF, but the current methodological reliance on fear discrimination paradigms in human research prevents direct assessment of mechanisms of safety learning that are distinct from those of fear learning. As we discuss below, the use of single-cue paradigms that conceptualize safety learning independently of fear learning has the potential to reveal unique clinically relevant applications for the study and treatment of OGF and anxiety.

\section{Clinical Implications of Safety Learning}

Some evidence (see Table 1 for summary of known effects of explicit safety learning from animal and human studies) suggest that safety learning has the potential to ameliorate anxiety by reducing both innate and learned fear (Hendry, 1967; Pollak et al., 2008; Rogan et al., 2005; Sangha et al., 2013; Tolin, 2019). In research with rodents, for example, the presence of safety signals has anxiolytic effects such that upon successful completion of safety learning, mice showed a significant reduction of depressive-like behaviors (e.g., decreased immobility in the forced-swim test) and innate anxiety (e.g., more time spent exploring the open arms of the elevatedplus maze, Pollak et al., 2008; more time exploring the center of an open field, Nahmoud et al., 2020) and better fear discrimination learning (Nahmoud et al., 2020). More research is needed to delineate the nature and magnitude of these effects (e.g., Are there enduring effects on anxious behaviors?).

In contrast with the animal literature, few studies to date have examined the direct impact of single-cued safety learning on human anxiety (for an exception, see Pollak, Rogan, et al., 2010). Thus, the field is limited in its ability to use converging methodologies to identify and examine mechanisms underlying the impact of safety signals on OGF and anxiety and to clarify clinical implications. Such knowledge has the potential to yield important clinical insights, similar to the translation of research on fear extinction and inhibitory learning into the development of exposure-based therapies (Craske et al., 2014; Öst, 1989).

Because safety learning increases attention to safety cues, there is potential for unique reduction of anxiety-related OGF via promotion of active coping and reward-focused cognition and behavior that may ameliorate anxiety, which is distinct from extinction but just as crucial (Kong et al., 2014; Meyer et al., 2019; Tolin, 2019). The findings from animal studies (Pollak et al., 2008; Sangha et al., 2013, 2020; Nahmoud et al., 2020) provide crucial initial evidence that safety learning may induce some of the behavioral and neural alterations that are similar to what pharmacological antidepressants would produce but using different pathways. Moreover, safety learning may have relevance for the prevention and remediation of anxiety-related avoidance, as anxiety-related OGF elicits avoidance of innocuous events or objects that may resemble threat (Dymond et al., 2012; Lommen et al., 2017; Nahmoud et al., 2020). For example, in one study, training subjects to

Table 1 Known effects of explicit safety learning (i.e., the presence of safety signals) from animal and human studies

\begin{tabular}{|c|c|}
\hline Behavioral effects of safety learning & Neurobiological effects of safety learning \\
\hline - Reward effects (Rogan et al., 2005; Sangha et al., 2013) & - Increase in BDNF Expression (Pollak et al., 2008) \\
\hline - Positive affect ("shelter-like" affect; Rogan et al., 2005) & - Regulation of amygdala miRNA expression (Ronovsky et al., 2019) \\
\hline $\begin{array}{l}\text { - Increase in explorative behavior in novel environments } \\
\text { (Pollak et al., 2008, Nahmoud et al., 2020) }\end{array}$ & - Reduction in spine size on synapses of the lateral amygdala (Ostroff et al., 2010) \\
\hline $\begin{array}{l}\text { - Increase in movement in the forced swim test } \\
\text { (Kong et al., 2014; Pollak et al., 2008) }\end{array}$ & $\begin{array}{l}\text { - Unique involvement of the posterior insular cortex during stress } \\
\text { mitigation (Christianson et al., 2008, 2011) }\end{array}$ \\
\hline $\begin{array}{l}\text { - Mitigate the consequences of intermittent stressors } \\
\text { (e.g., shock; Christianson et al., 2011, 2012) }\end{array}$ & $\begin{array}{l}\text { - Reduction in activity in the amygdala and increased activity in the dorsolateral } \\
\text { prefrontal cortex measured via diffusion tensor imaging (Pollak, Rogan, et al., 2010) }\end{array}$ \\
\hline
\end{tabular}


discriminate between threat and nonthreat (i.e., training to spot subtle differences in shape and size of stimuli thereby promoting perceptual discrimination) reduced maladaptive, anxietyrelated avoidance of nonthreat stimuli measured via behavioral responses to escape or avoid a potential US during each presentation of CS- and generalization stimuli (e.g., safety cue and cues that perceptually resemble the CS-; Lommen et al., 2017). This remediation of avoidance has clinical relevance given the role of behavioral and cognitive avoidance in the promotion and maintenance of anxiety by preventing individuals from confronting sources of fear or anxiety and reevaluating maladaptive cognitions and behaviors (Clark, 1999; Craske et al., 2014).

An important direction for future clinical research is to examine the association among safety learning, OGF, and other cognitive processes related to anxiety, such as intolerance of uncertainty (IU; Aikins \& Craske, 2001; Dugas et al., 1998; Ladouceur et al., 1997; Lissek et al., 2014b; Morriss et al., 2016). IU reflects exaggerated discomfort with the uncertain possibility that something aversive might occur. IU is inflated in clinical anxiety. Some have argued that IU is specifically characterized by disruptions in the assessment of safety relative to threat, such as exaggerated assessment of cost and probability of threat, hypervigilance to threat, and impaired safety signal processing (Grupe \& Nitschke, 2013). They further argue that intolerance of uncertainty may reciprocally dampen one's ability to evaluate accurately and to discriminate threat from nonthreat, thus exacerbating anxiety-related OGF. In line with this, studies using fear discrimination paradigms document that OGF is directly associated with IU (Aikins \& Craske, 2001; Ladouceur et al., 1997). In this line of work, a generalization gradient placing CS+ and CS- at each end of a spectrum with generalization stimuli (GS) that progressively become similar to $\mathrm{CS}+$ and CS- were used to assess OGF (e.g., a series of shapes gradually increasing in size; Armony et al., 1997; Lissek et al., 2008, 2010; Lissek et al., 2014b; Morriss et al., 2016). Subjects with low IU showed low OGF (i.e., stronger discrimination between threat and nonthreat), whereas those with high IU showed high OGF (Morriss et al., 2016). These findings mirror patterns of exaggerated OGF associated with anxiety (Lissek et al., 2014b; Lissek \& Grillon, 2015).

The role of cue salience is another component that could be examined through safety learning as an experimental paradigm that may be applicable for clinical research, as clinical anxiety is closely associated with impairments in learning cues that indicate safety (Jovanovic et al., 2012; Lissek et al., 2009). One approach is to enhance the salience of the safety CS by accompaniment of an additional cue, which in turn enhances allocation of attention to the safety CS, possibly increasing the strength of the association regarding the unpaired relationship between the CS and US. For example, in a typical safety learning experiment, there is uncertainty in the timing of when the US will be presented (i.e., random interstimulus-interval). This uncertainty can be anxiogenic and thus more clearly demarcating the period of safety with additional cues (a tone or visual stimulus) minimizes uncertainty and creates a greater sense of predictability (or smaller prediction error).

As depicted in Figure 2, our working model describes the effects of the presence of safety signals on anxiety via its impact on OGF by considering the learning context (e.g., cue salience) and individual differences in trait anxiety. First, the model posits that due to uncertainty during safety learning (e.g., the unpairing of CS and US leads to ambiguity as to when the threat will be present), and the presence of foreground conditioning, increasing salience of the safety CS may boost attention to the safe cue, thus more effectively reducing OGF. When OGF is reduced, the ability to discriminate cued threat from nonthreat is concomitantly enhanced (Jovanovic et al., 2012). The model further posits that the link between OGF and state anxiety will be influenced by anxietyrelated traits and individual differences, with greater state anxiety emerging from high OGF among relatively high trait anxious individuals.

Building on this model, we developed a set of three singlecued paradigms that directly vary the salience of the safety cue. This allows for a more targeted identification of the parameters that impact the strength of safety learning and is amenable to research with both human and animals. As described in Figure 3, these three single-cued safety learning

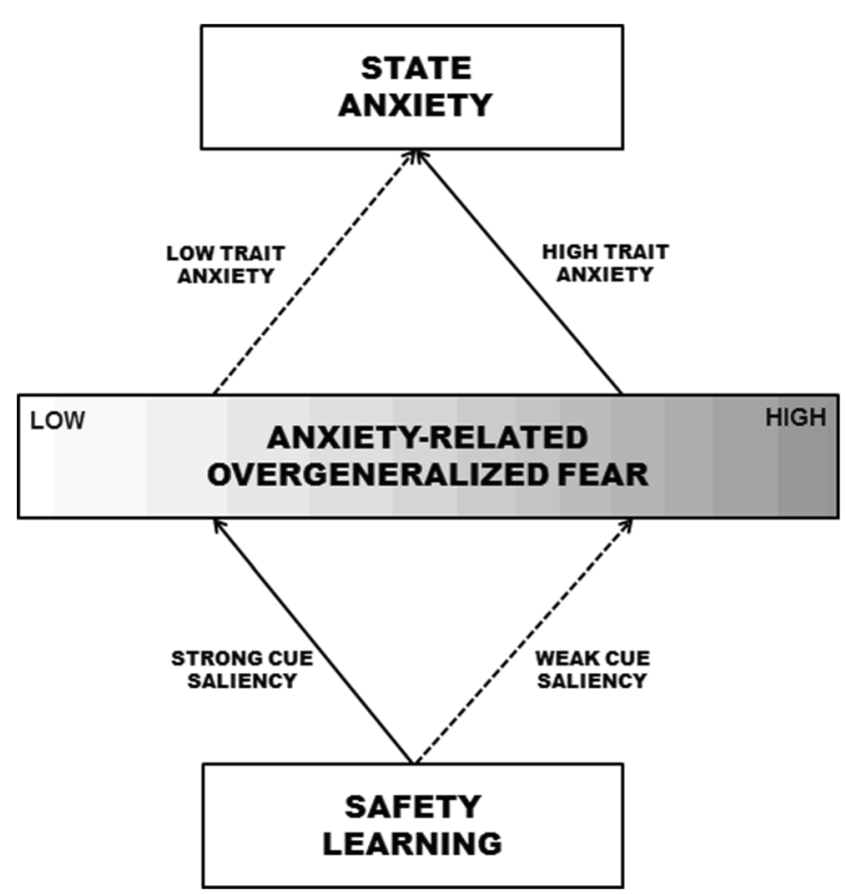

Fig. 2 Model positing the effect of safety learning on anxiety-related OGF and its effects on state anxiety while integrating consideration of cue salience and individual differences in trait anxiety. 
paradigms can be compared to a single-cued fear learning paradigm in a single experiment. In these paradigms, only one type of CS is presented, signaling either the presence (fear learning) or absence (safety learning) of shock during the cue presentation period. Whereas the "Safety Learning 1" condition does not manipulate salience, "Safety Learning 2" includes an auditory cue that co-terminates with the safety CS, and "Safety Learning 3" includes an auditory cue that cooccurs with the onset of the safety CS to boost its salience. The timing of the auditory cues makes either the end of the no shock/safe period ("Safety Learning 2") or the beginning of the no shock/safe period ("Safety Learning 3") relatively salient.

In a recent study using a between-subjects design, we employed these three single-cued safety paradigms in comparison with a single-cued fear paradigm in a strain of highanxiety phenotype mice. Results showed that mice in the safety paradigm, compared with the fear paradigm, evidenced lower levels of anxiety-like behaviors (e.g., less freezing and more exploration in novel environment) and improved discrimination learning of novel CS- stimuli. This effect was most prominent when salience of safety onset was boosted (Safety Learning 3; Nahmoud et al., 2020). These results document the impact of the presence of safety signals, and the importance of signal salience, on anxiety-related behaviors and threat-nonthreat discrimination, an index of OGF. Findings also show the promise of single-cued experimental paradigms that systematically vary the parameters of safety

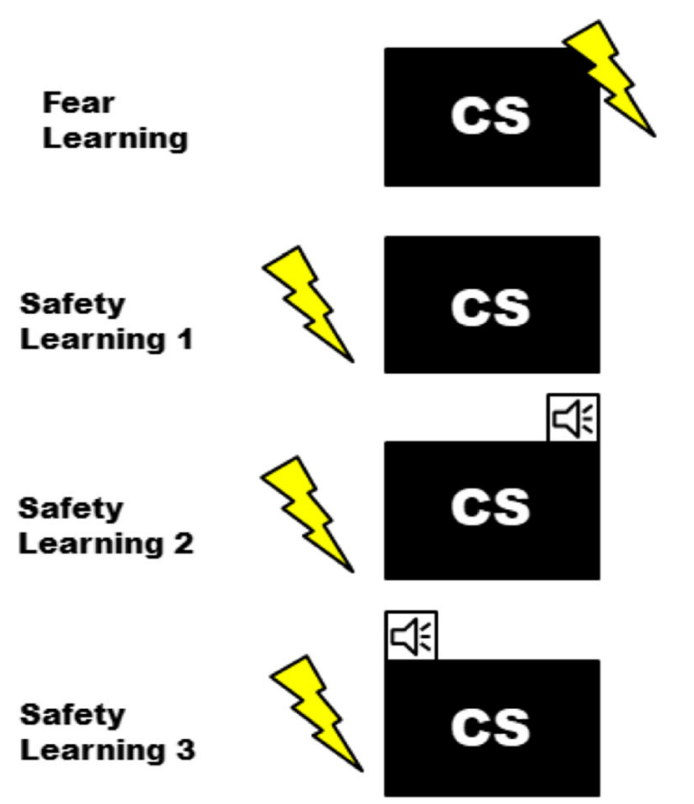

Fig. 3 Differences in CS-US schedule in fear learning vs. safety learning conditions (with or without an additional cue to add saliency). For human subjects, the CS was a visual cue (e.g., a colored shape) and the US was electrical stimulation. An auditory cue either co-terminated ("safety learning 2") or co-occurred ("safety learning 3") with the CS to add saliency. learning for advancing translational studies of OGF in humans and animals.

Importantly, consistent with the idea that feature negative learning, such as safety learning (i.e., cues signal the absence of shock), in combination with foreground conditioning is particularly sensitive to individual differences and difficult to learn compared with fear learning. The safety learning conditions overall showed more variability and weaker acquisition (Crowell \& Bernhardt, 1979). Such preliminary evidence suggests that variability in response to safety learning would differ between those with relatively high or low levels of OGF and/or trait anxiety. Furthermore, training effective safety learning in high-anxiety individuals by using more salient safety cues may prove to be a beneficial therapeutic approach to lower OGF and increase discrimination (Nahmoud et al., 2020).

In summary, the use of single-cued safety learning paradigms, such as those emerging from our working model, compared with fear learning, has the potential to clarify clinically meaningful mechanisms and individual differences in OGF as they relate to anxiety. Early evidence suggests that the presence of safety signals can ameliorate anxiety by reducing both innate and learned fear and boost activity in reward-related circuits. Furthermore, clinically relevant mechanisms of anxiety, such as avoidance and IU, also may be affected by safety learning. More research is needed to understand the specific effects that safety learning might have on these anxiety-related processes, which in turn could directly inform the development of more precise and personalized intervention approaches.

\section{Conclusions}

Methodological innovations in the translational study of safety learning can further our knowledge of anxiety-related OGF and foster the development of novel treatment approaches. Past studies document that the presence of safety signals, compared with fear learning, may uniquely elicit positive affect, contain rewarding properties (Pollak et al., 2008; Rogan et al., 2005; Sangha et al., 2013, 2020) that are associated with distinct underlying neural circuitry (Kong et al., 2014; Meyer et al., 2019; Ostroff et al. 2010; Sangha et al., 2013, 2014), and have direct anxiolytic and antidepressant effects (Nahmoud et al., 2020; Pollak et al., 2008). Yet, only few studies, especially in human research, conceptualize safety learning as an active learning mechanism independent of fear learning, leaving a significant empirical and methodological gap in the literature. A critical goal for future research is to use single-cued paradigms and examine contextual learning effects to study safety learning as both distinct from and related to fear learning and inhibitory learning (Huckleberry et al., 2016; Mohammadi et al., 2014; Nahmoud et al., 2020; 
Rogan et al., 2005; Sangha et al., 2013, 2014, 2020). This will allow not only the study of active and unique safety learning mechanisms in anxiety-related OGF but will provide more translational bridges with the animal literature in accordance with a growing body of literature emphasizing the importance of aligning cross-species methodologies (Haaker et al., 2019; Meyer et al., 2019). Furthermore, while anxiety-related OGF has been studied for decades in clinical contexts, the specific biological, cognitive, and behavioral substrates underlying OGF and its role in the etiology, maintenance, and remediation of anxiety remain elusive. Understanding the links between individual differences in safety and threat signal detection and more downstream and clinically characteristic behaviors related to anxiety-related OGF are crucial future empirical questions. Much more also must be understood about neurobiological mechanisms underlying safety learning, which in turn could yield important insights into clinical applications and the refinement of current treatment approaches.

Acknowledgments This work was supported by National Institute of Mental Health Grants R56MH111700 and RF1MH120846 (to T.D.T.), and R01MH118441 (to E.L.) and by the National Center for Advancing Translational Sciences Grant TR000457 and PSC-CUNY Enhanced Award (\#62796-00-50 to E.L.), and PSC-CUNY Award (\#61413-00-49 to T.D.T), jointly funded by The Professional Staff Congress and The City University of New York.

Financial Disclosures Ms. Cho, Dr. Likhtik, and Dr. Dennis-Tiwary reported no financial interests or potential conflicts of interest. Open Practices Statement None of the data or materials for the experiments reported are available, and none of the experiments was preregistered.

\section{References}

Aikins, D. E., \& Craske, M. G. (2001). Cognitive theories of generalized anxiety disorder. Psychiatric Clinics of North America, 24(1), 57-74.

Aizenberg, M. \& Geffen, M.N. (2013). Bidirectional effects of aversive learning on perceptual acuity are mediated by the sensory cortex. Nature Neuroscience, 16, 994-996.

Armony, J. L., Servan-Schreiber, D., Romanski, L. M., Cohen, J. D., \& LeDoux, J. E. (1997). Stimulus generalization of fear responses: effects of auditory cortex lesions in a computational model and in rats. Cerebral Cortex (New York, NY: 1991), 7(2), 157-165.

Asok, A., Kandel, E. R., \& Rayman, J. B. (2018). The neurobiology of fear generalization. Frontiers in Behavioral Neuroscience, 12.

Bar-Haim, Y., Lamy, D., Pergamin, L., Bakermans-Kranenburg, M. J., \& Van Ijzendoorn, M. H. (2007). Threat-related attentional bias in anxious and nonanxious individuals: a meta-analytic study. Psychological bulletin, 133(1), 1.

Beck, J. S. (1964) Cognitive Therapy: Basics and Beyond, New York: Guildford Press.

Besnard, A., \& Sahay, A. (2016). Adult hippocampal neurogenesis, fear generalization, and stress. Neuropsychopharmacology, 41(1), 24.

Böcker, K. B., Baas, J. M., Kenemans, J. L., \& Verbaten, M. N. (2004). Differences in startle modulation during instructed threat and selective attention. Biological Psychology, 67(3), 343-358.
Bublatzky, F., \& Schupp, H. T. (2012). Pictures cueing threat: brain dynamics in viewing explicitly instructed danger cues. Social Cognitive and Affective Neuroscience, 7(6), 611-622.

Bucci, D. J., Saddoris, M. P., \& Burwell, R. D. (2002). Contextual fear discrimination is impaired by damage to the postrhinal or perirhinal cortex. Behavioral Neuroscience, 116(3), 479.

Burgos-Robles, A., Kimchi, E. Y., Izadmehr, E. M., Porzenheim, M. J., Ramos-Guasp, W. A., Nieh, E. H., ... \& Anandalingam, K. K. (2017). Amygdala inputs to prefrontal cortex guide behavior amid conflicting cues of reward and punishment. Nature Neuroscience, 20(6), 824 .

Buzsáki, G., \& Wang, X. J. (2012). Mechanisms of gamma oscillations. Annual Review of Neuroscience, 35, 203-225.

Buzzell, G. A., Richards, J. E., White, L. K., Barker, T. V., Pine, D. S., \& Fox, N. A. (2017). Development of the error-monitoring system from ages 9-35: Unique insight provided by MRI-constrained source localization of EEG. Neuroimage, 157, 13-26.

Bystritsky, A. (2006). Treatment-resistant anxiety disorders. Molecular Psychiatry, 11(9), 805.

Cha, J., Greenberg, T., Carlson, J. M., DeDora, D. J., Hajcak, G., \& Mujica-Parodi, L. R. (2014). Circuit-wide structural and functional measures predict ventromedial prefrontal cortex fear generalization: implications for generalized anxiety disorder. Journal of Neuroscience, 34(11), 4043-4053.

Christianson, J. P., Benison, A. M., Jennings, J., Sandsmark, E. K., Amat, J., Kaufman, R. D., ... \& Barth, D. S. (2008). The sensory insular cortex mediates the stress-buffering effects of safety signals but not behavioral control. Journal of Neuroscience, 28(50), 13703-13711.

Christianson, J. P., Fernando, A. B., Kazama, A. M., Jovanovic, T., Ostroff, L. E., \& Sangha, S. (2012). Inhibition of fear by learned safety signals: a mini-symposium review. Journal of Neuroscience, 32(41), 14118-14124.

Christianson, J. P., Jennings, J. H., Ragole, T., Flyer, J. G., Benison, A. M., Barth, D. S., ... \& Maier, S. F. (2011). Safety signals mitigate the consequences of uncontrollable stress via a circuit involving the sensory insular cortex and bed nucleus of the stria terminalis. Biological Psychiatry, 70(5), 458-464.

Clark, D. M. (1999). Anxiety disorders: Why they persist and how to treat them. Behaviour Research and Therapy, 37, S5-S27.

Concina, G., Cambiaghi, M., Renna, A., \& Sacchetti, B. (2018). Coherent activity between the prelimbic and auditory cortex in the slowgamma band underlies fear discrimination. Journal of Neuroscience, 38(39), 8313-8328.

Craske, M. G., Rauch, S. L., Ursano, R., Prenoveau, J., Pine, D. S., \& Zinbarg, R. E. (2009). What is an anxiety disorder?. Depression and Anxiety, 26(12), 1066-1085.

Craske, M. G., Treanor, M., Conway, C. C., Zbozinek, T., \& Vervliet, B. (2014). Maximizing exposure therapy: an inhibitory learning approach. Behaviour Research and Therapy, 58, 10-23.

Crowell, C. R., \& Bernhardt, T. P. (1979). The feature-positive effect and sign-tracking behavior during discrimination learning in the rat. Animal Learning \& Behavior, 7(3), 313-317.

Cuthbert, B. N., Schupp, H. T., Bradley, M. M., Birbaumer, N., \& Lang, P. J. (2000). Brain potentials in affective picture processing: Covariation with autonomic arousal and affective report. Biological Psychology, 52(2), 95-111.

Davis, M., Falls, W. A., \& Gewirtz, J. (2000). Neural systems involved in fear inhibition: Extinction and conditioned inhibition. In Contemporary issues in modeling psychopathology (pp. 113-141). Springer, Boston, MA.

Denefrio, S., Myruski, S., Mennin, D., Dennis-Tiwary, T.A. (2019). When neutral is not neutral: Neurophysiological evidence for reduced discrimination between aversive and non-aversive information in generalized anxiety disorder. Motivation and emotion, 43, 325-338. 
Do-Monte, F. H., Quinones-Laracuente, K., \& Quirk, G. J. (2015). A temporal shift in the circuits mediating retrieval of fear memory. Nature, 519(7544), 460-463.

Dugas, M. J., Gagnon, F., Ladouceur, R., \& Freeston, M. H. (1998). Generalized anxiety disorder: A preliminary test of a conceptual model. Behaviour Research and Therapy, 36(2), 215-226.

Duits, P., Cath, D. C., Lissek, S., Hox, J. J., Hamm, A. O., Engelhard, I. M., ... \& Baas, J. M. (2015). Updated meta-analysis of classical fear conditioning in the anxiety disorders. Depression and Anxiety, 32(4), 239-253.

Dunsmoor, J. E., \& Paz, R. (2015). Fear generalization and anxiety: behavioral and neural mechanisms. Biological Psychiatry, 78(5), 336-343.

Dymond, S., Dunsmoor, J. E., Vervliet, B., Roche, B., \& Hermans, D. (2015). Fear generalization in humans: systematic review and implications for anxiety disorder research. Behavior Therapy, 46(5), 561582.

Dymond, S., Schlund, M. W., Roche, B., De Houwer, J., \& Freegard, G. P. (2012). Safe from harm: learned, instructed, and symbolic generalization pathways of human threat-avoidance. PLoS One, 7(10), e47539.

Eisenberg, D. \& Lipson, S. K. (2017). The healthy minds study 20162017 data report. Ann Arbor, MI: Author. Retrieved from http:// healthymindsnetwork.org

El-Bar, N., Laufer, O., Yoran-Hegesh, R., \& Paz, R. (2017). Overgeneralization in youth with anxiety disorders. Social Neuroscience, 12(1), 76-85.

Eysenck, M. W. (1979). Anxiety, learning, and memory: A reconceptualization. Journal of Research in Personality, 13(4), 363-385.

Fani, N., King, T. Z., Jovanovic, T., Glover, E. M., Bradley, B., Choi, K., ... \& Ressler, K. J. (2012). White matter integrity in highly traumatized adults with and without post-traumatic stress disorder. Neuropsychopharmacology, 37(12), 2740-2746.

Gazendam, F. J., Kamphuis, J. H., \& Kindt, M. (2013). Deficient safety learning characterizes high trait anxious individuals. Biological Psychology, 92(2), 342-352.

Girardeau, G., Inema, I., \& Buzsáki, G. (2017). Reactivations of emotional memory in the hippocampus-amygdala system during sleep. Nature Neuroscience, 20(11), 1634

Giustino, T. F., \& Maren, S. (2015). The role of the medial prefrontal cortex in the conditioning and extinction of fear. Frontiers in Behavioral Neuroscience, 9, 298.

Gould T.J., Bevins R.A. (2012) Context Conditioning. In: Seel N.M. (eds) Encyclopedia of the Sciences of Learning. Springer, Boston, MA. https://doi.org/10.1007/978-1-4419-1428-6_1029

Greenberg, T., Carlson, J. M., Cha, J., Hajcak, G., \& Mujica-Parodi, L. R. (2013). Ventromedial prefrontal cortex reactivity is altered in generalized anxiety disorder during fear generalization. Depression and Anxiety, 30(3), 242-250.

Grosso, A., Santoni, G., Manassero, E., Renna, A., \& Sacchetti, B. (2018). A neuronal basis for fear discrimination in the lateral amygdala. Nature Communications, 9(1), 1-12.

Grunfeld, I. S., Likhtik, E. (2018). Mixed selectivity encoding and action selection in the prefrontal cortex during threat assessment. Current Opinions in Neurobiology, 49, 108-115.

Grupe, D. W., \& Nitschke, J. B. (2013). Uncertainty and anticipation in anxiety: an integrated neurobiological and psychological perspective. Nature Reviews Neuroscience, 14(7), 488-501.

Guo, N., Soden, M. E., Herber, C., Kim, M. T., Besnard, A., Lin, P., .. \& \& Sahay, A. (2018). Dentate granule cell recruitment of feedforward inhibition governs engram maintenance and remote memory generalization. Nature medicine, 24(4), 438.

Haaker, J., Lonsdorf, T. B., Schümann, D., Menz, M., Brassen, S., Bunzeck, N., ... \& Kalisch, R. (2015). Deficient inhibitory processing in trait anxiety: Evidence from context-dependent fear learning, extinction recall and renewal. Biological Psychology, 111, 65-72.
Haaker, J., Maren, S., Andreatta, M., Merz, C. J., Richter, J., Richter, S. H., ... \& Seidenbecher, T. (2019). Making translation work: Harmonizing cross-species methodology in the behavioural neuroscience of Pavlovian fear conditioning. Neuroscience \& Biobehavioral Reviews, 107, 329-345.

Haddad, A. D., Pritchett, D., Lissek, S., \& Lau, J. Y. (2012). Trait anxiety and fear responses to safety cues: Stimulus generalization or sensitization?. Journal of Psychopathology and Behavioral Assessment, 34(3), 323-331.

Hajcak, G., Castille, C., Olvet, D. M., Dunning, J. P., Roohi, J., \& Hatchwell, E. (2009). Genetic variation in brain-derived neurotrophic factor and human fear conditioning. Genes, Brain and Behavior, $8(1), 80-85$.

Hajcak, G., Dunning, J. P., \& Foti, D. (2009). Motivated and controlled attention to emotion: time-course of the late positive potential. Clinical Neurophysiology, 120(3), 505-510.

Han, J. H., Yiu, A. P., Cole, C. J., Hsiang, H. L., Neve, R. L., \& Josselyn, S. A. (2008). Increasing CREB in the auditory thalamus enhances memory and generalization of auditory conditioned fear. Learning \& Memory, 15(6), 443-453.

Hendry, D. P. (1967). Conditioned inhibition of conditioned suppression. Psychonomic Science, 9(5), 261-262.

Huckleberry, K. A., Ferguson, L. B., \& Drew, M. R. (2016). Behavioral mechanisms of context fear generalization in mice. Learning \& Memory, 23(12), 703-709.

Indovina, I., Robbins, T. W., Núñez-Elizalde, A. O., Dunn, B. D., \& Bishop, S. J. (2011). Fear-conditioning mechanisms associated with trait vulnerability to anxiety in humans. Neuron, 69(3), 563-571.

Iordanova, M. D., Killcross, A. S., \& Honey, R. C. (2007). Role of the medial prefrontal cortex in acquired distinctiveness and equivalence of cues. Behavioral Neuroscience, 121(6), 1431.

Ito, W., \& Morozov, A. (2019). Prefrontal-amygdala plasticity enabled by observational fear. Neuropsychopharmacology, 44(10), 1778-1787.

Izadmehr, E.M., Porzenheim, M.J., Ramos-Guasp, W.A., Nieh, E.H., et al. (2017). Amygdala inputs to prefrontal cortex guide behavior amid conflicting cues of reward and punishment. Nature Neuroscience, 20, 824-835.

Jin, J., \& Maren, S. (2015). Fear renewal preferentially activates ventral hippocampal neurons projecting to both amygdala and prefrontal cortex in rats. Scientific Reports, 5, 8388.

Johansen, J. P., Tarpley, J. W., LeDoux, J. E., \& Blair, H. T. (2010). Neural substrates for expectation-modulated fear learning in the amygdala and periaqueductal gray. Nature Neuroscience, 13(8), 979.

Jovanovic, T., Kazama, A., Bachevalier, J., \& Davis, M. (2012). Impaired safety signal learning may be a biomarker of PTSD. Neuropharmacology, 62(2), 695-704.

Karalis, N., Dejean, C., Chaudun, F., Khoder, S., Rozeske, R. R., Wurtz, H., ... \& Herry, C. (2016). 4-Hz oscillations synchronize prefrontalamygdala circuits during fear behavior. Nature Neuroscience, 19(4), 605.

Kessler, R. C., Petukhova, M., Sampson, N. A., Zaslavsky, A. M., \& Wittchen, H. U. (2012). Twelve-month and lifetime prevalence and lifetime morbid risk of anxiety and mood disorders in the United States. International Journal of Methods in Psychiatric Research, 21(3), 169-184.

Kim, W.B. \& Cho, J.H. (2017). Synaptic targeting of double-projecting ventral CA1 hippocampal neurons to the medial prefrontal cortex and basal amygdala. Journal of Neuroscience, 37, 4868-4882.

Klavir, O., Genud-Gabai, R., \& Paz, R. (2013). Functional connectivity between amygdala and cingulate cortex for adaptive aversive learning. Neuron, 80(5), 1290-1300.

Klavir, O., Prigge, M., Sarel, A., Paz, R., \& Yizhar, O. (2017). Manipulating fear associations via optogenetic modulation of amygdala inputs to prefrontal cortex. Nature neuroscience, 20(6), 836 . 
Kong, E., Monje, F. J., Hirsch, J., \& Pollak, D. D. (2014). Learning not to fear: neural correlates of learned safety. Neuropsychopharmacology, 39(3), 515-527.

Kroenke, K., Spitzer, R. L., Williams, J. B., Monahan, P. O., \& Löwe, B. (2007). Anxiety Disorders in Primary Care: Prevalence, Impairment, Comorbidity, and Detection Anxiety Disorders in Primary Care. Annals of Internal Medicine, 146(5), 317-325.

Ladouceur, R., Talbot, F., \& Dugas, M. J. (1997). Behavioral expressions of intolerance of uncertainty in worry: Experimental findings. Behavior Modification, 21(3), 355-371.

Letzkus, J. J., Wolff, S. B., Meyer, E. M., Tovote, P., Courtin, J., Herry, C., \& Lüthi, A. (2011). A disinhibitory microcircuit for associative fear learning in the auditory cortex. Nature, 480(7377), 331-335.

Likhtik, E., \& Paz, R. (2015). Amygdala-prefrontal interactions in (mal) adaptive learning. Trends in Neurosciences, 38(3), 158-166.

Likhtik, E., Stujenske, J. M., Topiwala, M. A., Harris, A. Z., \& Gordon, J. A. (2014). Prefrontal entrainment of amygdala activity signals safety in learned fear and innate anxiety. Nature Neuroscience, 17(1), 106113

Lis, S., Thome, J., Kleindienst, N., Mueller-Engelmann, M., Steil, R., Priebe, K., .. \& \& Bohus, M. (2020). Generalization of fear in posttraumatic stress disorder. Psychophysiology, 57(1), e13422

Lissek, S. (2012). Toward an account of clinical anxiety predicated on basic, neurally mapped mechanisms of Pavlovian fear learning: the case for conditioned overgeneralization. Depression and Anxiety, 29(4), 257-263.

Lissek, S., Biggs, A. L., Rabin, S. J., Cornwell, B. R., Alvarez, R. P., Pine, D. S., \& Grillon, C. (2008). Generalization of conditioned fearpotentiated startle in humans: experimental validation and clinical relevance. Behaviour Research and Therapy, 46(5), 678-687.

Lissek, S., Bradford, D. E., Alvarez, R. P., Burton, P., Espensen-Sturges, T., Reynolds, R. C., \& Grillon, C. (2014a). Neural substrates of classically conditioned fear-generalization in humans: a parametric fMRI study. Social Cognitive and Affective Neuroscience, 9(8), 1134-1142.

Lissek, S., Kaczkurkin, A. N., Rabin, S., Geraci, M., Pine, D. S., \& Grillon, C. (2014b). Generalized anxiety disorder is associated with overgeneralization of classically conditioned fear. Biological Psychiatry, 75(11), 909-915.

Lissek, S., \& Grillon, C. (2015). Overgeneralization of conditioned fear in the anxiety disorders. Zeitschrift für Psychologie/Journal of Psychology.

Lissek, S., Powers, A. S., McClure, E. B., Phelps, E. A., Woldehawariat, G., Grillon, C., \& Pine, D. S. (2005). Classical fear conditioning in the anxiety disorders: a meta-analysis. Behaviour Research and Therapy, 43(11), 1391-1424.

Lissek, S., Rabin, S., Heller, R. E., Lukenbaugh, D., Geraci, M., Pine, D. S., \& Grillon, C. (2010). Overgeneralization of conditioned fear as a pathogenic marker of panic disorder. American Journal of Psychiatry, 167(1), 47-55.

Lissek, S., Rabin, S. J., McDowell, D. J., Dvir, S., Bradford, D. E., Geraci, M., ... \& Grillon, C. (2009). Impaired discriminative fearconditioning resulting from elevated fear responding to learned safety cues among individuals with panic disorder. Behaviour Research and Therapy, 47(2), 111-118.

Liu, J., Likhtik, E., Shereen, D., Dennis, T. A., \& Casaccia, P. (2020). White Matter Plasticity in Anxiety: Disruption of Neural Network Synchronization during Threat-Safety Discrimination. Frontiers in Cellular Neuroscience, 14, 351.

Lommen, M. J., Duta, M., Vanbrabant, K., de Jong, R., Juechems, K., \& Ehlers, A. (2017). Training discrimination diminishes maladaptive avoidance of innocuous stimuli in a fear conditioning paradigm. PloS one, 12(10), e0184485.

Lonsdorf, T. B., Menz, M. M., Andreatta, M., Fullana, M. A., Golkar, A., Haaker, J., ... \& Drexler, S. M. (2017). Don't fear 'fear conditioning': Methodological considerations for the design and analysis of studies on human fear acquisition, extinction, and return of fear. Neuroscience \& Biobehavioral Reviews.

Lovibond, P. F., Chen, S. X., Mitchell, C. J., \& Weidemann, G. (2013). Competition between an avoidance response and a safety signal: evidence for a single learning system. Biological Psychology, 92(1), 9-16.

Luo, R., Uematsu, A., Weitemier, A., Aquili, L., Koivumaa, J., McHugh, T. J., \& Johansen, J. P. (2018). A dopaminergic switch for fear to safety transitions. Nature Communications, 9(1), 1-11.

Maren, S., Aharonov, G., \& Fanselow, M. S. (1997). Neurotoxic lesions of the dorsal hippocampus and Pavlovian fear conditioning in rats. Behavioural Brain Research, 88(2), 261-274.

Maren, S., \& Fanselow, M. S. (1996). The amygdala and fear conditioning: has the nut been cracked?. Neuron, 16(2), 237-240.

Maren, S., Phan, K. L., \& Liberzon, I. (2013). The contextual brain: implications for fear conditioning, extinction and psychopathology. Nature Reviews Neuroscience, 14(6), 417.

Mathews, A., \& MacLeod, C. (2005). Cognitive vulnerability to emotional disorders. Annual Review of Clinical Psychology, 1, 167-195.

McHugh, T. J., Jones, M. W., Quinn, J. J., Balthasar, N., Coppari, R., Elmquist, J. K., ... \& Tonegawa, S. (2007). Dentate gyrus NMDA receptors mediate rapid pattern separation in the hippocampal network. Science, 317(5834), 94-99.

McNally, G. P., \& Cole, S. (2006). Opioid receptors in the midbrain periaqueductal gray regulate prediction errors during pavlovian fear conditioning. Behavioral Neuroscience, 120(2), 313.

Meyer, H. C., \& Bucci, D. J. (2014). The contribution of medial prefrontal cortical regions to conditioned inhibition. Behavioral Neuroscience, 128(6), 644.

Meyer, H. C., Odriozola, P., Cohodes, E. M., Mandell, J. D., Li, A., Yang, R., ... \& Lee, F. S. (2019). Ventral hippocampus interacts with prelimbic cortex during inhibition of threat response via learned safety in both mice and humans. Proceedings of the National Academy of Sciences, 116(52), 26970-26979.

Milad, M. R., Orr, S. P., Pitman, R. K., \& Rauch, S. L. (2005). Context modulation of memory for fear extinction in humans. Psychophysiology, 42(4), 456-464.

Mogg, K., Bradley, B., Miles, F., \& Dixon, R. (2004). Brief report time course of attentional bias for threat scenes: testing the vigilanceavoidance hypothesis. Cognition and Emotion, 18(5), 689-700.

Mohammadi, M., Bergado-Acosta, J. R., \& Fendt, M. (2014). Relief learning is distinguished from safety learning by the requirement of the nucleus accumbens. Behavioural Brain Research, 272, 40 45.

Moita, M.A., Rosis, S., Zhou, Y., LeDoux, J.E., Blair, H.T. (2004). Putting fear in its place: remapping of hippocampal place cells during fear conditioning. Journal of Neuroscience, 24, 7015-7023.

Morriss, J., Macdonald, B., \& Van Reekum, C. M. (2016). What is going on around here? Intolerance of uncertainty predicts threat generalization. PloS One, 11(5), e0154494.

Moscarello, J. M., \& Maren, S. (2018). Flexibility in the face of fear: Hippocampal-prefrontal regulation of fear and avoidance. Current Opinion in Behavioral Sciences, 19, 44-49.

Nahmoud, I., Vasquez, J. G., Cho, H., Dennis-Tiwary, T., \& Likhtik, E. (2020). Salient Safety Conditioning Improves Novel Discrimination Learning. Behavioural Brain Research, 112907.

Nelson, B. D., Weinberg, A., Pawluk, J., Gawlowska, M., \& Proudfit, G. H. (2015). An event-related potential investigation of fear generalization and intolerance of uncertainty. Behavior Therapy, 46(5), 661-670.

O'Reilly, R. C., \& Rudy, J. W. (2001). Conjunctive representations in learning and memory: principles of cortical and hippocampal function. Psychological Review, 108(2), 311.

Öst, L. G. (1989). One-session treatment for specific phobias. Behaviour Research and Therapy, 27(1), 1-7. 
Ostroff, L. E., Cain, C. K., Bedont, J., Monfils, M. H., \& LeDoux, J. E. (2010). Fear and safety learning differentially affect synapse size and dendritic translation in the lateral amygdala. Proceedings of the National Academy of Sciences, 107(20), 9418-9423.

Ozawa, T., Ycu, E. A., Kumar, A., Yeh, L. F., Ahmed, T., Koivumaa, J., \& Johansen, J. P. (2017). A feedback neural circuit for calibrating aversive memory strength. Nature neuroscience, 20(1), 90-97.

Padilla-Coreano, N., Bolkan, S.S., Pierce, G.M., Blackman, D.R., Hardin, W.D., Garcia-Garcia, A.L., et al. (2016). Direct ventral hippocampal-prefrontal input is required for anxiety-related neural activity and behavior. Neuron, 89, 857-866.

Pavlov, I. P. (1927). Conditional reflexes: An investigation of the physiological activity of the cerebral cortex.

Phelps, E. A., Delgado, M. R., Nearing, K. I., \& LeDoux, J. E. (2004). Extinction learning in humans: role of the amygdala and vmPFC. Neuron, 43(6), 897-905.

Phelps, E. A., \& LeDoux, J. E. (2005). Contributions of the amygdala to emotion processing: from animal models to human behavior. Neuron, 48(2), 175-187.

Pittig, A., Treanor, M., LeBeau, R. T., \& Craske, M. G. (2018). The role of associative fear and avoidance learning in anxiety disorders: Gaps and directions for future research. Neuroscience \& Biobehavioral Reviews.

Pollak, D. D., Monje, F. J., \& Lubec, G. (2010). The learned safety paradigm as a mouse model for neuropsychiatric research. Nature Protocols, 5(5), 954-962.

Pollak, D. D., Monje, F. J., Zuckerman, L., Denny, C. A., Drew, M. R., \& Kandel, E. R. (2008). An animal model of a behavioral intervention for depression. Neuron, 60(1), 149-161.

Pollak, D. D., Rogan, M. T., Egner, T., Perez, D. L., Yanagihara, T. K., \& Hirsch, J. (2010). A translational bridge between mouse and human models of learned safety. Annals of Medicine, 42(2), 127-134.

Quirk, G. J., Garcia, R., \& González-Lima, F. (2006). Prefrontal mechanisms in extinction of conditioned fear. Biological Psychiatry, 60(4), 337-343.

Raber, J., Arzy, S., Bertolus, J. B., Depue, B., Haas, H. E., Kangas, M., ... \& Mouly, A. M. (2019). Current understanding of fear learning and memory in humans and animal models and the value of a linguistic approach for analyzing fear learning and memory in humans. Neuroscience \& Biobehavioral Reviews, 105, 136-177.

Ray, M. H., Hanlon, E., \& McDannald, M. A. (2018). Lateral orbitofrontal cortex partitions mechanisms for fear regulation and alcohol consumption. PloS one, 13(6), e0198043.

Ray, M. H., Russ, A. N., Walker, R. A., \& McDannald, M. A. (2020). The nucleus accumbens core is necessary to scale fear to degree of threat. Journal of Neuroscience, 40(24), 4750-4760.

Redondo, R. L., Kim, J., Arons, A. L., Ramirez, S., Liu, X., \& Tonegawa, S. (2014). Bidirectional switch of the valence associated with a hippocampal contextual memory engram. Nature, 513(7518), 426.

Rescorla, R. A. (1967). Pavlovian conditioning and its proper control procedures. Psychological Review, 74(1), 71.

Rogan, M. T., Leon, K. S., Perez, D. L., \& Kandel, E. R. (2005). Distinct neural signatures for safety and danger in the amygdala and striatum of the mouse. Neuron, 46(2), 309-320.

Ronovsky, M., Zambon, A., Cicvaric, A., Boehm, V., Hoesel, B., Moser, B. A., ... \& Pollak, D. D. (2019). A role for miR-132 in learned safety. Scientific Reports, 9(1), 1-16.

Rozeske, R. R., Jercog, D., Karalis, N., Chaudun, F., Khoder, S., Girard, D., ... \& Herry, C. (2018). Prefrontal-periaqueductal grayprojecting neurons mediate context fear discrimination. Neuron, 97(4), 898-910.

Sangha, S., Chadick, J. Z., \& Janak, P. H. (2013). Safety encoding in the basal amygdala. Journal of Neuroscience, 33(9), 3744-3751.
Sangha, S., Diehl, M. M., Bergstrom, H. C., \& Drew, M. R. (2020). Know safety, no fear. Neuroscience \& Biobehavioral Reviews.

Sangha, S., Robinson, P. D., Greba, Q., Davies, D. A., \& Howland, J. G. (2014). Alterations in reward, fear and safety cue discrimination after inactivation of the rat prelimbic and infralimbic cortices. Neuropsychopharmacology, 39(10), 2405-2413.

Sarlitto, M. C., Foilb, A. R., \& Christianson, J. P. (2018). Inactivation of the ventrolateral orbitofrontal cortex impairs flexible use of safety signals. Neuroscience, 379, 350-358.

Schechtman, E., Laufer, O., \& Paz, R. (2010). Negative valence widens generalization of learning. Journal of Neuroscience, 30(31), 10460 10464.

Seidenbecher, T., Laxmi, T. R., Stork, O., \& Pape, H. C. (2003). Amygdalar and hippocampal theta rhythm synchronization during fear memory retrieval. Science, 301(5634), 846-850.

Sep, M. S., Steenmeijer, A., \& Kennis, M. (2019). The relation between anxious personality traits and fear generalization in healthy subjects: A systematic review and meta-analysis. Neuroscience \& Biobehavioral Reviews, 107, 320-328.

Struyf, D., Zaman, J., Hermans, D., \& Vervliet, B. (2017). Gradients of fear: How perception influences fear generalization. Behaviour Research and Therapy, 93, 116-122.

Struyf, D., Zaman, J., Vervliet, B., \& Van Diest, I. (2015). Perceptual discrimination in fear generalization: Mechanistic and clinical implications. Neuroscience \& Biobehavioral Reviews, 59, 201-207.

Stujenske, J. M., Likhtik, E., Topiwala, M. A., \& Gordon, J. A. (2014). Fear and safety engage competing patterns of theta-gamma coupling in the basolateral amygdala. Neuron, 83(4), 919-933.

Taub, A. H., Perets, R., Kahana, E., \& Paz, R. (2018). Oscillations synchronize amygdala-to-prefrontal primate circuits during aversive learning. Neuron, 97(2), 291-298.

Tolin, D. F. (2019). Inhibitory learning for anxiety-related disorders. Cognitive and Behavioral Practice, 26(1), 225-236.

Venkataraman, A., Brody, N., Reddi, P., Guo, J., Rainnie, D. G., \& Dias, B. G. (2019). Modulation of fear generalization by the zona incerta. Proceedings of the National Academy of Sciences, 116(18), 90729077.

Walker, R. A., Wright, K. M., Jhou, T. C., \& McDannald, M. A. (2020). The ventrolateral periaqueductal grey updates fear via positive prediction error. European Journal of Neuroscience, 51(3), 866-880.

Wang, M. E., Wann, E. G., Yuan, R. K., Álvarez, M. M. R., Stead, S. M., \& Muzzio, I. A. (2012). Long-term stabilization of place cell remapping produced by a fearful experience. Journal of neuroscience, 32(45), 15802-15814.

Weinberger, N. M. (2007). Associative representational plasticity in the auditory cortex: a synthesis of two disciplines. Learning \& Memory, 14(1-2), 1-16.

Wigestrand, M. B., Schiff, H. C., Fyhn, M., LeDoux, J. E., \& Sears, R. M. (2017). Primary auditory cortex regulates threat memory specificity. Learning \& Memory, 24(1), 55-58.

Wild, J. M., \& Blampied, N. M. (1972). Hippocampal lesions and stimulus generalization in rats. Physiology \& Behavior, 9(4), 505-511.

Wood, K. C., Blackwell, J. M., \& Geffen, M. N. (2017). Cortical inhibitory interneurons control sensory processing. Current Opinion in Neurobiology, 46, 200-207.

Zelikowsky, M., Hersman, S., Chawla, M. K., Barnes, C. A., \& Fanselow, M. S. (2014). Neuronal ensembles in amygdala, hippocampus, and prefrontal cortex track differential components of contextual fear. Journal of Neuroscience, 34(25), 8462-8466.

Publisher's note Springer Nature remains neutral with regard to jurisdictional claims in published maps and institutional affiliations. 